Methods Data from systematic reviews, dedicated methodological experimental studies and qualitative studies nested within behaviour change trials will be presented.

Results Study findings indicate: (1) the existence of previously unrecognised recruitment effects on behaviour; (2), that assessment reactivity is a widespread form of contamination in behaviour change trials; (3) that randomisation itself may induce behaviour change; and (4) that these types of effects are highly unlikely to be additive to the effect of behavioural interventions, thus leading to biased estimates of effectiveness.

Conclusions The scale of the problem, the implications for reviews of existing trials, and the reasons for the lack of development of study in this crucial area for behaviour change trials are discussed. Hypotheses and study designs are proposed to guide new research which seeks to quantify problems with existing practice in the design and conduct of trials.

\section{P1-40 EXPERIENCE OF DISCRIMINATION IS A SIGNIFICANT ASSOCIATE OF MENTAL ILL-HEALTH IN IRISH TRAVELLERS. FINDINGS FROM THE ALL IRELAND TRAVELLER HEALTH STUDY}

doi:10.1136/jech.2011.142976c.33

C McGorrian, B Quirke, C Kelleher,* All Ireland Traveller Health Study Team. UCD School of Public Health, Physiotherapy and Population Science, Dublin, Ireland

Introduction Traveller are an indigenous minority in Ireland, with poorer life expectancy and health status than the general population. We describe here the self-reported burden of mental ill-health and its associates.

Methods A census survey of all Travellers was undertaken, with 8492 enumerated families ( $80 \%$ response rate). A random subset of 1796 adults completed a health survey. Peer researchers employed a novel oral-visual computer data-collection methodology. Age and sex-adjusted logistic regression models were fitted, with one or more days of mental ill-health in the last month as the dependent variable. Results Overall $39 \%$ of men (225/580) and $41 \%$ of women (495/ 1211) reported mental ill-health, increasing with age $(p=0.001)$. In a multivariable model, factors associated with increased odds of mental ill-health were poorer physical health (OR 4.7, 95\% CI 3.3 to 6.8), being unable to enjoy usual activities (OR 17.2, 95\% CI 11.7 to 25.2 ), regular alcohol consumption (OR 1.5, 95\% CI 1.0 to 2.3), agreeing that drugs are a community problem (OR 1.8, 95\% CI 1.3 to 2.6), that nomadism is important (OR $1.5,95 \%$ CI 1.0 to 2.2 ), and increasing experience of discrimination (OR 1.1 per 1-point increase in scale, $95 \%$ CI 1.0 to 1.1). Factors associated with reduced odds were male sex (OR $0.7,95 \%$ CI 0.44 to 0.95 ), rural vs urban living (OR $0.5,95 \%$ CI 0.4 to 0.8 ), and social supports (OR 0.84 per $10 \%$ increase in scale, $95 \%$ CI 0.75 to 0.97 . AUROC was 0.92 .

Conclusions This novel study comprehensively profiles associations of mental ill-health in a vulnerable minority community.

\section{P1-41 THE INFLUENCE OF FOREST LANDSCAPE DESIGN ON HUMAN-TICK CONTACT AS AN EFFORT TO PREVENT EXPOSURE TO TICK-BORNE DISEASES}

doi:10.1136/jech.2011.142976c.34

C Meha. ${ }^{*}$ UMR 8185 Espaces, Nature et Culture, CNRS/ Paris IV/ Paris 8, Paris, France

Background Reducing exposure to ticks is currently the most effective method of prevention of Lyme borreliosis, which appears to pose a new public health problem in heavily urbanised areas. The analysis of contacts between the routes that people adopt in forests (where ticks live) and the spaces and environments considered to be of risk (the most suitable habitat for ticks) constitutes a privileged avenue of study. There is a need to study these spatial dynamics, as well as to study ways in which it is possible to minimise risk via the landscape and design.

Methods Two databases were created, one related to ticks that can transmit the infection and the other to trajectories of forest users. The first was fed by samples collected in the Sénart forest (France) and the second gathered descriptive data on volume and characteristics of human flow through the forest area.

Results All the data have been entered into a GIS database. A characterisation of the busiest portions of routes in relation to data on tick populations densities and distribution (and, in fact, a characterisation of individual vulnerabilities on the type of sociodemographic profile associated with these portions) has then been conducted and has enable us to model human exposure to ticks according to the locations visited by users.

Conclusions Various actions related to forest management will be discussed with the forest officers such as, for example, the relocation or closure of some trails, or the changing of points of attractions for users in the forest.

\section{P1-42 ASSOCIATIONS OF AREA DEPRIVATION OVER THE LIFECOURSE AND PHYSICAL CAPABILITY IN MID-LIFE: FINDINGS FROM THE 1946 BRITISH BIRTH COHORT}

doi:10.1136/jech.2011.142976c.35

${ }^{1,2}{ }^{2}$ T Murray, ${ }^{*}{ }^{3} Y$ Ben-Shlomo, ${ }^{4} \mathrm{H}$ Southall, ${ }^{4} \mathrm{P}$ Aucott, ${ }^{3} \mathrm{~K}$ Tilling, ${ }^{2} \mathrm{~J}$ Guralnik, ${ }^{1} \mathrm{D}$ Kuh, ${ }^{1} \mathrm{R}$ Hardy. ${ }^{1} \mathrm{MRC}$ Unit for Lifelong Health and Ageing, London, UK; ${ }^{2} \mathrm{NIH} / \mathrm{NIA} /$ Laboratory of Epidemiology, Demography, and Biometry, Bethesda, Maryland, USA; ${ }^{3}$ Department of Social Medicine, University of Bristol, Bristol, UK; ${ }^{4}$ Great Britain Historical GIS Project, Department of Geography, University of Portsmouth, Portsmouth, UK

Background Recent work has shown that factors across the whole of life influence physical capability in later life. Therefore, when investigating area socioeconomic effects on physical capability, area exposures should be assessed across the lifecourse to take account changes in residence and secular trends of an area.

Methods Using data from the MRC National Survey of Health and Development, we examined the relationship between area low social class (per cent partly- or un-skilled of all occupied in a local government district) at ages 4,26 , and 53 years [residence linked to census data for years 1951, 1971, and 2001) with objective measures of physical capability (grip strength, standing balance and chair rise time) at age 53 years.

Results After adjustment for area at other years, a higher area low social class at 4 and 53 years was associated with decline in mean balance time of $4.4 \%$ (95\% CI 0.6 to 8.1 ) and $7.6 \%$ (3.6 to 11.6 ), respectively, but only area at age 53 with higher chair rise time [mean change $1.8 \%$ (95\% CI 0.0 to 3.6$)$ at 53 years. Associations were reduced but still apparent after adjustment for individual occupational social class at the same three ages. There were no significant associations between area and grip strength.

Conclusions For the first time, our study shows that living in a socioeconomically deprived area in early and later life adversely affect some measures of physical capability in mid-life. Future work is needed to explore potential mechanisms of area effects by age and physical capability measures.

\section{P1-43 BRIDGING ELECTRICAL DATA ENTRY SHEET AND STATISTICAL SOFTWARE BY STANDARD DATA MODEL}

doi:10.1136/jech.2011.142976c.36

M Okada. * Department of Epidemiology, University of Tsukuba, Tsukuba, Ibaraki, Japan

Introduction Interoperability of collected dataset has been distress for researchers. Introduction of database management software made some improvements, however, still we need many of 
craftworks to convert and clean up original dataset before the beginning of statistical analysis. To improve procedure of data management by raising interoperability of dataset, we technically examined a standard-based object-oriented data model for a common database between data entry system and statistical analysing system, and estimated how the process of data management was changed with the new data model.

Methods We adopted the Archetypes data model, which is a standard of ISO 13606, as the storage for collected dataset and the $\mathrm{R}$ language and environment for statistical computing as the statistical software. An example dataset was sampled from a cohort study. We simulated to develop an exclusive data entry sheet for the study. To estimate effect of introducing Archetypes database, we enumerated operations which will be required to build the system, input, review, clean, transfer, and analysis the example dataset. The complexity of each operation was estimated. For control, same estimation was performed on a system with traditional database.

Results Archetypes approach was expected to require more complicated procedures to build the data entry system than traditional approach, however, more software components was expected to reusable between other datasets. Both approaches were expected to require similar number of operations to manage datasets.

Conclusion For electrical data collection of epidemiological study, introduction of standardised data model might lead to efficient development of data entry system.

\section{P1-44 APPLICATION OF A NOVEL METHOD TO ESTIMATE INCIDENCE OF HEPATITIS C AMONG INJECTING DRUG USERS IN SCOTLAND}

doi:10.1136/jech.2011.142976c.37

\begin{abstract}
${ }^{1}$ Norah Palmateer, ${ }^{*}{ }^{2}$ Elizabeth Allen, ${ }^{2}$ Avril Taylor, ${ }^{1,3}$ Sharon Hutchinson, ${ }^{1}$ David Goldberg. ${ }^{1}$ Health Protection Scotland, Glasgow, UK; ${ }^{2}$ University of the West of Scotland, Paisley, UK; ${ }^{3}$ Department of Mathematics and Statistics, University of Strathclyde, Glasgow, UK
\end{abstract}

Background Prevalence of the hepatitis C virus (HCV) among injecting drug users (IDUs) in Scotland is high. Through an HCV Action Plan, the Scottish Government has invested significantly in harm reduction interventions with the goal of reducing HCV transmission among IDUs. In evaluating the effectiveness of interventions, estimates of the incidence, rather than the prevalence, of HCV are essential. The traditional approach to measuring incidence-follow-up of a cohort of uninfected individuals to measure seroconversion-can, however, be costly and suffer high participant attrition rates. We report the first large-scale, national application of a novel method designed to determine incidence of HCV using a cross-sectional design.

Methods During 2008-2009, IDUs were recruited from needle exchange services, completed an interviewer-administered questionnaire and provided a dried blood spot for anonymous HCVantibody (anti-HCV) and HCV-RNA testing. Incident infections were defined as individuals who were anti-HCV negative and HCVRNA positive.

Results Prevalence of anti-HCV was 55\% among the 2563 participants. Among anti-HCV negative respondents, twenty-one were HCV-RNA positive, yielding an incidence estimate of 12.1 per 100 person-years (corresponding to a viraemic pre-seroconversion window period of 51 days). Individuals currently receiving methadone had lower odds of incident infection (adjusted OR 0.15; 95\% CI 0.035 to 0.68 ) relative to those on methadone in the previous 6 months (but not currently).
Conclusions This approach to measuring incidence will allow us to gauge the impact of preventive interventions associated with the HCV Action Plan.

\section{P1-45 NOVEL APPLICATION OF LOT QUALITY ASSURANCE SAMPLING: AS A QUALITY CONTROL TOOL IN CENSUS VERIFICATION}

doi:10.1136/jech.2011.142976c.38

${ }^{1} \mathrm{~B}$ K Patro, ${ }^{2} \mathrm{~S}$ Saha, ${ }^{2} \mathrm{~K}$ Anand, ${ }^{2} \mathrm{~S} \mathrm{~K}$ Kapoor. ${ }^{1} \mathrm{P}$ GIMER school of public health Chandigarh, India; ${ }^{2}$ Centre for community medicine, AIIMS, New Delhi, India

Introduction Lot Quality Assurance Sampling (LOAS) was developed as technique for quality assurance in industrial production. The use of LOAS in health sciences is limited to coverage evaluations of different health activities such as immunisation coverage, antenatal care coverage, elimination of neonatal tetanus and coverage iodised salt and others. LOAS has been accepted as rapid epidemiological survey method. The present study was carried out to explore the feasibility of using LOAS in Census Verification.

Methods Comprehensive Rural Health Services Project (CRHSP) located in northern India, caters to a population of 70000 spread in 28 villages. All the individuals in the project area have been assigned a 10 digit unique identification number. Every year a health census is carried out to update the computerised health management information system by multi-purpose health workers. Quality control measure of such activity amounts cross verification of records of $25 \%$ population by the medical officer. The whole process takes 2 months time. LOAS was applied as a quality control measure for census operation. Village was considered as a Lot. $25 \%$ individual IDs were selected randomly from each village for quality check of demographic and health records. Allowable error was fixed at $5 \%$. If the error exceeded the limit of $5 \%$ the Lot was considered to be rejected and the whole census process was repeated.

Results Four villages were rejected upon using LOAS. The total duration of census operation was similar to previous method.

Conclusion LOAS can be used a scientific and robust method of quality control of census verification.

\section{P1-46 DIAGNOSTIC RADIATION EXPOSURE AND BREAST CANCER RISK IN BRCA1/2 MUTATION CARRIERS IN THE GENE-RAD- RISK STUDY}

doi:10.1136/jech.2011.142976c.39

${ }^{1} \mathrm{~A}$ Pijpe, ${ }^{*}{ }^{2} \mathrm{~N}$ Andrieu, ${ }^{3} \mathrm{D}$ Easton, ${ }^{4} \mathrm{~A}$ Kesminiene, ${ }^{5} \mathrm{E}$ Cardis, ${ }^{6} \mathrm{Genepso}$ study group, ${ }^{6} \mathrm{C}$ Nogues, ${ }^{3}$ Embrace study group, ${ }^{3} \mathrm{~S}$ Peock, ${ }^{3} \mathrm{D}$ Frost, ${ }^{1}$ Hebon study group, ${ }^{7} \mathrm{P}$ Manders, ${ }^{4} \mathrm{I}$ Thierry-Chef, ${ }^{8} \mathrm{D}$ Goldgar, ${ }^{1} \mathrm{M}$ Hauptmann, ${ }^{1} \mathrm{M}$ Rookus, ${ }^{1} \mathrm{~F}$ van Leeuwen. ${ }^{1}$ Netherlands Cancer Institute, Amsterdam, The Netherlands; ${ }^{2}$ Institut Curie, Paris, France; ${ }^{3}$ University of Cambridge, Cambridge, UK; ${ }^{4}$ IARC, Lyon, France; ${ }^{5}$ CREAL, Barcelona, Spain; ${ }^{6}$ Centre René Huguenin, Saint-Cloud, France; ${ }^{7}$ Radboud University Nijmegen Medical Centre, Nijmegen, The Netherlands; ${ }^{8}$ University of Utah, School of Medicine, Salt Lake City, USA

Introduction BRCA1/2 mutation carriers might be more sensitive to ionising radiation due to impaired DNA repair mechanisms.

Methods A retrospective European collaborative cohort study (GENE-RAD-RISK) of 1993 female BRCA1/2 mutation carriers was performed using self-reported exposure to diagnostic radiation. Risk of breast cancer was estimated using a weighted Cox proportional hazards model with cumulative radiation exposure from diagnostic procedures as a time-dependent variable lagged by 5 years.

Results are not presented in the abstract due to a publication embargo. 